\title{
Conference Proceedings of the Third International Vivano Conference held from 19th - 21st March 2014 in Nuremberg, Germany
}

D URING a two-day conference international speakers presented their experiences with negative pressure wound therapy in chronic wounds but also in cases of third- and fourth-degree burns, in complex surgical interventions of the abdominal region, in open fractures as well as in thoracic and cardiac surgery. The this year's conference focused on questions whether negative pressure wound therapy (NPWT) resulted in wound healing more often and more quickly than standard wound therapy, for which indications can NPWT be used and how should the cost-benefit ratio be evaluated.

\section{PlenARY SEsSion}

\section{Clinical and economic benefits of NPWT T. Banasiewicz}

Department of General, Endocrynological Surgery and Gastrointestinal Oncology, Poznan Medical University of Medical Sciences, Poznań, Poland.

Purpose: The evaluation of the clinical and economic benefits of negative pressure wound therapy (NPWT).

Methods: Thirty-seven patients treated for complicated lower open abdomen wounds between 2009-2012 were included, of whom 20 underwent standard therapy involving abdominal closure using zipping/temporary sutures while 17 received NPWT. The NPWT group was further subdivided into patients with NPWT initiation $\leq 5$ days after diagnosis/admission (7) and those $>5$ days (10). The two main treatment groups and NPWT subgroups were respectively homogeneous.

Results: NPWT more effectively reduced complications compared to standard therapy, with shorter hospital (26 vs. 43 days) and intensive care stays (13 vs. 22 days). The total treatment costs per NPWT patient were 33\% less than per standard-treatment patient. Both the hospital and patientspecific costs were less for NPWT treatment. The mortality rate was lower with NPWT (18 vs. 45\%) while the cost per saved life was $55 \%$ less compared to standard treatment. Initiating NPWT earlier further improved the patient condition, with shorter hospital (22.7 vs. 28.7 days) and intensive care stays ( 12 vs. 14 days), lower total cost $(9,803 €$ vs. $13,095 €)$, lower mortality (14 vs. $20 \%$ ) and a $30 \%$ reduction in the cost per saved life.
Conclusion: Although there was an insufficient number of patients to demonstrate a statistical effect, the results indicated that healing using NPWT appeared more effective at less cost, leading to lower mortality and a better quality of life. NPWT should be initiated immediately on diagnosis/admission rather than as a last resort.

\section{Prevention strategies in arthroplasty using negative pressure wound therapy}

\section{R. Becker}

General Orthopedics and Rheumatology, Eduardus Hospital, Cologne, Germany.

Purpose: To improve the procedure in implant infections by anticipation rather than as a response.

Methods: Joint punctures are performed prior to revision surgery and should infection be found, a two-stage revision is performed. The prosthesis is explanted together with radical debridement of the infected material and jet lavage wound rinsing. A spacer is inserted at the joint to allow movement. The operating time within the wound should not exceed 10 min. When preserving hip implants, it is preferable to replace all interchangeable components. Aspiration is performed as routine, applying negative pressure wound therapy (NPWT), preferably excising the foil to avoid leakage and system failure. The wound should be dry to avoid clotting in the foam and it is unnecessary to tighten the foil because this may lead to upper skin surface damage. Systemic antibiotic treatment (6-8 weeks) is initiated, later using oral antibiotics. Regular clinical checks are important to monitor clinical parameters. Antibiotic therapy is discontinued 7 days prior to performing three consecutive weekly joint punctures under sterile conditions with long-term incubation of a minimum of 10 days to test for infection, because low-level infection does not normally appear until after 5-6 days. After three consecutive infectionfree punctures, reimplantation is performed. A 1-year followup is recommended to ensure no prosthesis loosening.

Conclusion: Despite reduced comfort during treatment, this new concept lowers costs, requires less NPWT, allows better patient handling, makes it easier to plan operations and reduces wound complications, including further revisions/operations. 


\section{ABDOMINAL SESSION}

\section{Vacuum-assisted laparostomy in the treatment of peritonitis and biliary fistula \\ V. N. Obolensky}

SBFEHC City Clinical Hospital No. 13 of the Department of Healthcare of Moscow, Russia; Russian National Research Medical University, Moscow, Russia.

Introduction: Negative pressure wound therapy (NPWT) was applied in laparostomy in a complicated wound with multiple origins.

Case description: A 35-year-old had undergone emergency abdominal surgery for a pancreas-penetrating duodenal ulcer, with bleeding from the head glands and vessels. Inflammatory infiltrate involving the hepatoduodenal ligament, common bile duct and gallbladder was found. Two-thirds of the stomach was resected, with duodenal stump closure and cholecystectomy. Remedial re-laparotomy was performed after 5 days for marked peritonitis symptoms, which showed pancreatic head necrosis and enzyme peritonitis. After 4 days, remedial relaparotomy was performed and within 6 days, marked biliary discharge from the lower wound-corner sutures occurred. Relaparotomy was repeated, with ongoing peritonitis diagnosed, with bile on postoperative adhesions and bile leakage from the cystic duct stump. The abdominal cavity was cleansed, the cystic duct stump fixated using TachoComb sponge and vacuum-assisted dressing installed in the peritoneal cavity using a disposable sterile VivanoMed abdominal kit attached to VivanoTec to apply NPWT. The patient was supplied after 2 days with an abdominal compression bandage over the vacuum-assisted dressings to become mobilised. The vacuumassisted dressing was removed after 2 days. Peritonitis was absent, drainage was installed in the cystic duct stump and the abdominal wall wound was sutured. Within 2 weeks laboratory parameters were normal and the bile leakage had ceased. Following drainage and stitches removal the patient was discharged, with no recurrence or wound complications at follow-up.

Conclusion: NPWT can be applied in laparostomy to treat complicated abdominal wounds with multiple origins.

\section{Negative pressure therapy in the treatment of enteroatmospheric fistulas}

\section{R. Škuta, Y. Mykyta, I Keher}

Clinic of General Surgery, Faculty Hospital, Trnava, Slovakia.

Introduction: Enteroatmospheric fistula (EAF) is a major complication of open abdomen (OA), leading to protein loss and sepsis with high mortality. We present a multiple-treatment approach including negative pressure wound therapy (NPWT).
Case description: A 42-year-old male with abdominal trauma after initial treatment at a district hospital was transferred to our hospital. Surgery showed diffuse purulent peritonitis, multiple small-intestine adhesions, transversostomy necrosis and left subphrenic abscess. Abscess evacuation, right hemicolectomy, terminal ileostomy and drainage were performed, leaving an OA. Despite multiple operations over 2 months, peritonitis and necrosis remained, and EAFs developed in the rigid and thickened small intestine. NPWT was applied to treat septic complications and for intestinal content derivation. After 12 NPWT applications, granulation had started and the wound reduced, however, the intestinal fistulas remained. A Foley catheter was inserted in the most proximal fistula and NPWT applied to reduce EAF secretion. NPWT was temporarily halted to reduce hypergranulation using boric acid. After 10 months and 105 operations, all six EAFs were removed by resection of two parts of the small intestine, and two primary entero-enteral anastomoses were created. The OA was closed using polypropylene netting and the skin cover reconstructed. This had healed after 3 months, with the small intestine working again, and the patient was discharged for home treatment with monthly ambulatory visits. The patient had full enteral intake without malnutrition, no abdominal-wall defect or fistula and a permanent ileostomy.

Conclusion: A multidisciplinary approach, including NPWT and long-term total parenteral nutrition led to successful healing.

\section{Combining prosthetic material and negative} pressure therapy in very large abdominal wall defect reconstruction

\section{Hutan}

II. Surgical Clinic of the Medical Faculty, Comenius University, Hospital of St. Cyril and Methodius, Bratislava, Slovakia.

Introduction: Standard reconstruction surgery cannot be performed when tissue transfer is prevented because of large abdominal tissue loss and massive wound infection. Negative pressure wound therapy (NPWT) was applied in combination with prosthetic material as a "salvage technique".

Cases descriptions Patient 1 required operation and small ventral hernia repair. The abdominal wall displayed necrotising fasciitis. Following resection, sepsis developed and multiple organ failure occurred, the patient required sedation and artificial ventilation. A large part of the abdominal wall of a second male (Patient 2) consisted of hernia sites, resulting in a large defect after resection. Patient 3 required surgery for a suspected gallbladder tumour.

Defects were covered using bi-faced polypropylene nets with a hydrophilic layer and NPWT was applied to accelerate granulation and prevent graft infection. Patient 1 underwent necrectomy and after 12 negative pressure changes granulation was sufficient for split-skin grafting. Three redressings controlled for the infection, and the patient recovered from 
sepsis. In Patient 2, sublay mesh with hydrophilic substance and NPWT were applied, however, part of the skin became necrotic. This was excised and the defect treated further using mesh and NPWT. Sufficient granulation tissue developed for split-skin grafting and the patient healed. Patient 3 required three polypropylene nets of $20 \mathrm{~cm}$ x $30 \mathrm{~cm}$. NPWT induced granulation, allowing split-skin grafting and eventual healing with very good abdominal wall functionality.

Conclusion: This "salvage technique" proved life-saving and minimised the complications resulting from the absence of an abdominal wall.

\section{Treatment of tertiary peritonitis using negative pressure therapy}

\section{S. Shlyapnikov, A. Demko, I. Batyrshin}

Severe Sepsis Care Center of The Science Research Institute for Emergency Care n.a. Djanelidze, Saint-Petersburg, Russia.

Introduction: Tertiary peritonitis causes the highest mortality in intraabdominal infections. We present a case study using negative pressure wound therapy (NPWT) to temporarily close a laparotomy wound during tertiary peritonitis treatment.

Case description: A 55-year-old male was admitted to the emergency department with polytrauma 1 hour after an assault, including abdominal hemoperitoneum and rupture of the ileal mesentery, ascending colon and caecum. The patient underwent a right hemicolectomy, a $150-\mathrm{cm}$ small-intestine resection with ileotransverse anastomosis and right pleural cavity drainage. After 7 days the patient dramatically worsened, with low arterial pressure and clinical signs of diffuse peritonitis. Leakage and rupture of the ileotransverse anastomosis were found. An open abscess into the peritoneal cavity and two perforated ulcers of the small intestine 30 and $50 \mathrm{~cm}$ from the Treitz ligament were present. Anastomosis resection and reconstruction were performed as well as suturing of the acute ulcers, abdominal debridement and NPWT application using a VivanoMed abdominal organ protection layer and foam in the wound, covered with hydrofilm and connected via a VivanoTec port to VivanoTec. Although the patient was stabilising after 3 days of NPWT, intestinal contents were observed in the Vivano container, with an acute ulcer of the anastomosis requiring relaparotomy. Resection anastomosis was performed, creating an end ileostomy, and NPWT was reapplied for 6 days until the peritonitis had resolved and the abdominal wall was closed.

Conclusion: NPWT is an effective, useful and safe method for temporary abdominal wall wound closure during relaparotomy treatment of tertiary peritonitis.

\section{SPECIAL INDICATIONS}

\section{Endoscopic vacuum therapy in the upper gastrointestinal tract}

\section{G. Laukötter}

Department of General and Visceral Surgery, University Medical Center, Münster, Germany.

Introduction: Major upper gastrointestinal (GI) tract leakages are life-threatening surgical complications. The gold standard treatment is endoscopic implantation of a flexible covered mesh stent. However, problems include a lack of drainage, stent migration, inadequate defect closure, granulation tissue ingrowth and scar strictures. Endoscopic vacuum therapy (EVT) was adapted for the upper GI tract, which we evaluated in 31 cases of upper GI tract perforations.

Methods: The indications for therapy were anastomotic insufficiency (23), iatrogenic oesophageal perforation (4), traumatic oesophageal perforation (1), Boerhaave's syndrome (2) and tumour perforation (1). The sponge was trimmed to the wall defect/wound cavity geometry, perforated with a metal rod connected to the gastric tube, the rod removed and the sponge fixed firmly to the distal end of the gastric tube using solid threads. This was attached parallel to the endoscope, using a loop at the end of the foam to the endoscopic grasper. The whole was inserted into the wall defect/wound cavity under direct vision and the tube diverted through the nose for connection to a vacuum pump run at a negative pressure of -120 to $-125 \mathrm{mmHg}$. The median EVT duration was 29 days (4-114 days), with eight endoscopic interventions (1-29) and a maximum of eight sponge changes.

Results: Complete closure occurred in $94 \%$ of cases, with no complications, therapy failure, or unintended defect enlargement during endoscopic examination or therapy. Drainage was possible with EVT, unlike in stenting.

Conclusion: EVT is applicable to nearly all possible upper GI-tract leakages, and may replace stent therapy.

\section{Negative pressure wound therapy in maxillofacial surgery}

\section{Niedzielska, M. Nowinski, K. Sciskala}

Department of Cranio-Maxillo-Facial and Oral Surgery, Medical University of Silesia, Poland.

Introduction: We present a case study of negative pressure wound therapy (NPWT) in cranio-maxillofacial surgery.

Case description: A 64-year-old female with squamous cell carcinoma of the mandible displayed complicated face and neck healing following oncological surgery. The patient developed marginal necrosis of the skin-muscle flap, purulent exudation, extraoral wound dehiscence, lymph accumulation 
in the lower neck and moderate peripheral inflammation. Necrotic tissue was removed, NPWT initiated using VivanoTec and Atrauman Ag, and prescribed antibiotics, anticoagulants and painkillers. Granulation had started by day 10, however, another problem appeared lower on the neck, to which the polyurethane foam was extended for simultaneous NPWT treatment. The lower wound was sutured on day 12. Infection was absent on day 15 and the patient discharged. Every 2 days, the vacuum dressing was changed, using Atrauman Ag, Sorbalgon and Hydrosorb gel. By day 23, the patient had no pain and the upper wound was granulated. NPWT was stopped, while Atrauman $\mathrm{Ag}$ and Sorbalgon were continued. Complete reepithelialisation occurred by day 61 . The patient was re-hospitalised on day 65 for partial reconstructive plate removal and flap reconstruction, applying NPWT and using Atrauman Ag for 2 days. Atrauman Ag and Aquacel Ag were applied for 11 days until wound dehiscence and infection were absent, and the patient discharged. The patient received painkillers for moderate pain and Hydrosorb gel dressing, changed every 2 days for 2 weeks. Complete healing was observed 1 week later.

Conclusion: NPWT will help to prepare patients for further treatment in complicated cranio-maxillofacial cases.

\section{Endoscopic negative pressure wound therapy in the upper gastrointestinal region}

\section{J. F. Lock ${ }^{1}$, S. Reimer ${ }^{2}$, M. Scheurlen ${ }^{2}$, C. T. Germer ${ }^{1}$}

1. Department of General-, Visceral-, Vascular- and Pediatric Surgery,

2. Department of Gastroenterology, University Hospital, Würzburg, Germany.

Introduction: The incidence of anastomotic leaks is relatively high in the upper gastrointestinal (GI) region, particularly after oesophagectomy and gastrectomy. We have adapted endoscopic therapy for rectal anastomotic leaks to the upper GI region, using a vacuum pump combined with a sufficiently sized collecting receptacle. Here we present five patients treated using this endoscopic negative pressure wound therapy (E-NPWT).

Case descriptions: One patient had suffered a spontaneous perforation of the oesophagus while the other four had postoperative leaks following oesophagogastrostomy (two), oesophagojejunostomy (one) and gastric fundus (one). The mean perforation diameter was $16 \pm 9 \mathrm{~cm}$. The gastric tube was placed through the nose, pulled out of the mouth and inserted into the sponge. The sponge was fixed to the endoscope, inserted orally and positioned intraluminally for small perforations or initially intracavitary for larger defects, switching to intraluminally when almost closed. A negative pressure of -120 to $-125 \mathrm{mmHg}$ was applied for a median of 10 days (1-56 days) and three E-NPWT sessions (1-17), depending on perforation size, with a sponge change every 3-4 days. Closure was achieved directly in four patients. One patient with a postoperative leak after oesophagogastrostomy developed a secondary fistula of the bronchi. E-NPWT was ceased and surgical treatment led to full recovery. Biliary secretion removal was optimal and no malfunctions occurred. All patients achieved normal mucosa and food intake.

Conclusion: E-NPWT appears to be the most effective therapy for upper GI leaks and is now the primary strategy for such patients in the department.

\section{TRAUMA SESSION}

\section{Negative pressure wound therapy treatment of} sternal wound infection following cardiac surgery: Vivano as the first line of therapy

\section{P. Zacek}

Department of Cardiac Surgery, Charles University Hospital, Hradec Kralove, Czech Republic

Purpose: We present an overview of our experience of the application of negative pressure wound therapy (NPWT) in the treatment of deep sternal wound infection following cardiac surgery.

Methods: When infection was evident, the wound was opened aggressively, together with wire and fluid material removal, to determine whether the problem went deeper, including a sternal hull split with some form of instability. Surgical toilette was applied as aggressively as possible, disinfecting agents were used and a silver-containing mesh covered the whole surface. The sponge was shaped to specific need. A deeper layer filled the gap between the sternum halves to prevent sternal-hull closure while a superficial layer filled the soft layer. A coverage sheet was placed over the whole wound and the suction hub applied. NPWT was initiated using the VivanoTec unit, with the first dressing change after 4/5 days and then normally twice weekly, dependent on the secretion and tissue appearance, using painkillers or injections rather than anaesthesia.

Results: From 2011-2013, 52 cardiac surgery patients with 335 exchanges (median 5 per patient) obtained full reconstruction of skin integrity without any mortality, and only six patients required rehospitalisation. One patient has since been lost, possibly because of hesitation over reopening.

Conclusion: NPWT can be readily applied to the longitudinal defects of sternotomy and to variable defect sizes. There should be no delay in wound opening and NPWT application, together with an aggressive surgical approach, including necrectomy. This should be combined with systemic therapy/topical chemotherapy, nutrition and psychological support. 


\section{Negative pressure treatment of a complex trauma with tibial open fracture}

\section{G. Noditi, S. Dragusanu}

Clinics of Plastic and Reconstructive Surgery "Austria House", Emergency Clinical County Hospital, Timisoara, Romania.

Introduction: We applied negative pressure wound therapy (NPWT) to a complex trauma with an open fracture.

Case description: A 29-year-old male was transferred to our clinic with a complex trauma and open fracture at the left tibial pillar previously fixed using a $\mathrm{K}$ wire, with fracture site contamination and bone necrosis. The patient received antibiotic therapy for 1 week. Contaminating elements and necrotic tissue were removed together with the primary osteosynthesis material. The tibia was stabilised using external fixation. Following debridement and application of a polyurethane sponge, dressing was placed over the defect and NPWT initiated using Vivano. After 3days there was very good granulation in the internal defect and re-epithelialisation at the skin-defect margin. Depending on the aspirate volume, further negative pressure was applied at 3 and 5 days. By day 14 , there was good granulation tissue progress. After 4 weeks, again depending on the aspirate volume, negative pressure was applied at 3 or 5 days. Granulation tissue covered the defect and re-epithelialisation of the margin was very good. After 6 weeks there was no microbial contamination. The defect was closed using skin grafts and a spacer introduced to protect the bone segments for 3-4 months. Subsequently, an autologous bone graft was performed. Finally, a bone reconstruction by free transfer fibular autology was planned.

Conclusion: NPWT provided good drainage, prevented contamination, accelerated granulation tissue formation of very good quality, allowing skin grafting and subsequent autologous bone grafting, and was comfortable for the patient.

\section{Does negative pressure wound therapy reduce wound infection?}

\section{Lenka Veverkova, Michal Reska, Jan Zak, Ivan Capov}

1st Surgical Department, St. Anne's Hospital Faculty of Medicine, Masaryk University, Brno, Czech Republic.

Purpose: A prospective study of 70 patients with infected wounds treated using negative pressure wound therapy (NPWT).

Method Seventy patients from January 2010 to December 2013 with a median age 62 years $(5-95 \%$ percentiles: $23-85$ years), with infected wounds of $8 \mathrm{~cm} * 5 \mathrm{~cm}$ to $38 \mathrm{~cm} *$ $35 \mathrm{~cm}$ and hospitalised because of wounds to the abdomen, chest, limb or sacral area were included in this prospective study. Material was obtained from the wound using a swab before, during and after NPWT to determine the level and profile of bacteria present.
Results: No mortalities occurred and the wounds healed accompanied by a reduced wound bacterial load, with an improvement achieved in $>20 \%$ of the cases, although Staphylococcus aureus and Escherichia coli remained present during and after NPWT treatment. Thus NPWT could be recommended for application to infected exuding wounds. Interestingly, NPWT treatment was age-dependent, with patients $\geq 60$ years compared to those of $<60$ years receiving significantly longer NPWT treatment (median 18 [4-45] days and 11 [4-34] days, respectively; $\mathrm{p}=0.034$ ), with more frequent dressing changes (median 4 and 3 days, respectively) and remained significantly longer in hospital (median 49 [15114] days and 29 [7-100] days, respectively; $p=0.003$ ).

Conclusion: NPWT can be used in a wide range of cases to reduce wound bacterial load. However, a positive cultivation result does not necessarily mean it leads to a clinical manifestation. Therefore, this prospective study is unable to provide a clear yes or no answer.

\section{The role of negative pressure wound therapy in thoracic surgery}

\section{Zs. Szentkereszty, I Takács, A. Enyedi, K. Szabó, Cs. Váradi, A. Csiszkó}

Institute of Surgery, Medical Health Science Center, University of Debrecen, Debrecen, Hungary.

Introduction: We review negative pressure wound therapy (NPWT) use in two indications for thoracic surgery: thoracic empyema and deep infected sternal wounds.

Methods: In thoracic empyema the thoracotomy was left open or open window thoracostomy was performed, the wound debrided, the cause of the empyema eliminated, the heart and lung covered with a non-adherent layer, the foam inserted, hermetic closure achieved using a hydrofilm and negative pressure was generally applied at -100 to $-125 \mathrm{mmHg}$, followed by primary closure or reconstructive surgery. The dressing was changed every 3-4 days under general anaesthesia or sedation and NPWT could be reapplied later as an outpatient. Complications, including pain, hypotension, tachycardia, bleeding and bronchopleural fistula, could be ameliorated by lowering the pressure. Treatment was 0.5-7.7 months. Treatment of a deep infected sternal wound involved antibiotic therapy, extensive debridement and reconstruction. NPWT preparation and treatment were similar to thoracic empyema, including the dressing changes, with a negative pressure of -100 to -130 $\mathrm{mmHg}$, decreased to $-75 \mathrm{mmHg}$ for complications. NPWT may be combined with other drainage therapy and can act as a "bridging therapy" from septic wound to plastic surgery. There was an indication for prophylactic NPWT in critically ill patients after cardiac surgery.

Results: NPWT was well tolerated in thoracic empyema and deep infected sternal wounds, with near $100 \%$ success and a high primary closure rate. 
Conclusion: Patients had an excellent quality of life and treatment was cost effective, with a shorter hospital stay, antibiotic therapy and outpatient therapy.

\section{Negative pressure wound therapy in high-energy soft-tissue injury management}

\section{Ali}

I Department of Orthopaedic and Trauma Surgery, Centre Hospitalier Régional d'Orléans, Hospital La Source, Orléans, France.

Introduction: We present our first experience using negative pressure wound therapy (NPWT) in high-energy softtissue injury management.

Case descriptions: A 54-year-old male presented with a high-velocity trauma to the left leg with a significant defect of the posterior thigh and associated nerve injury and contamination. Following rinsing and debridement, the skin flap was sutured. Skin necrosis and infection were found after the second dressing change on day 6. Following extensive debridement, NPWT was applied using the VivanoTec unit at $-75 \mathrm{mmHg}$, with extra skin protection provided by a thin hydrocolloid dressing around the edges. By day 6 there was rapid granulation, including covering the previously exposed tendon, and there was no infection. The patient was discharged and continued treatment at home. Complete healing was achieved by day 30 without grafting, with a full range of knee movement. The patient returned to work after 45 days. A 78 year-old male had suffered a large crush wound to the posterior of the left leg, with massive tissue damage and loss, and wound contamination. The wound was rinsed and debrided, and the skin flap sutured. After 6 days there was cutaneous and muscular necrosis. Following debridement, NPWT was initiated. There was rapid granulation to enable skin grafting. Complete wound healing occurred by day 45 , the patient returning to normal activities.

Conclusion: Both patients healed with no complications. Vivano has become a vital tool in high-energy soft-tissue injury management, reducing the wound closure time and hospital stay while improving the quality of life.

\section{BURNS}

Closure of third and fourth degree burns of the fingers, hand dorsum and forearm using negative pressure therapy

\section{Jurisic}

Surgery Department, General and County Hospital „Dr. J. Benčević”, Slavonski Brod, Croatia.

Introduction: Following third and fourth degree burns, structures are subject to drying, infection and necrosis, with the risk of amputation. Negative pressure wound therapy (NPWT) was applied to assist in wound closure management.

Case description: A 58-year-old female had suffered fourth degree burns to the underside of the left forearm and digits, and second and partial third degree injuries of the second digit. NPWT was initiated after primary wound debridement and digit escharectomy. Four weeks post-injury the whole fifth digit and the fourth digit from the proximal interphalangeal joint were amputated, covering the stumps with a skin flap created from these digits. After 4 weeks of NPWT, the significant amount of remaining exposed bone was debrided. The wound healed properly but was hard due to the gasteroenterocolitis, and a wound infection was treated systemically using tazocin. The vacuum was removed and the wound irrigated three times daily. Six weeks post-injury there was sufficient granulation without infection. A large mesh graft was applied and NPWT restarted. The patient self-discharged after 1 week, but returned 3 months post-injury with almost complete attachment. Arthrodesis was performed of the proximal interphalangeal joint of the unstable third digit, together with sequential debridement of the distal and proximal joints and of the middle part. The appearance was very good, opposition of the first and second digits was normal while wrist function was almost normal

Conclusion: NPWT in this burn produced lasting and durable skin with more natural coverage than flap surgery, without additional bulking procedures.

\section{Negative pressure treatment of a complex leg defect after electrical burn}

\section{Alexandru Ulici, Iulia Tevanov, Madalina Carp, Gabriel}

\section{A. Sterian, Catalin Nahoi}

Pediatric Orthopaedics, Bucharest, Romania.

Introduction: Severe limb burn injuries are difficult to treat and are at risk of amputation. We applied negative pressure wound therapy (NPWT) to a complex burn wound.

Case description: A 17-year-old male had suffered highvoltage burns, with muscle and tendon necrosis of the right leg lower extremity, which was excised. On transfer to our department, there was a major soft-tissue defect with tibial 
bone exposure in the lower half of the right leg and a smaller defect with exposed peroneal malleolus. The devitalised, exposed tibia was resected, the wound edges excised, the wound cleaned and covered using nanocrystalline silver wound dressing, a hydrocolloid dressing was used to protect the skin between the defects and wound edges, and a foam bridge linked both defects. Initial continuous-mode NPWT caused significant blood loss. NPWT was changed to intermittent mode and haemorrhaging ceased within 12 hours. Local cleaning, soft-tissue avivement and dressing changes under general anaesthesia were performed twice weekly for 6 weeks. Silver and intermittently silicone wound dressings controlled local infection and the patient received intravenous antibiotics. During the final stage of NPWT, the bone surface of the main defect remained partially exposed. This was covered using silicone dressing and silver dressing was applied to the granulated area. On completing NPWT, skin grafting was performed on both beds using a free skin graft from the ipsilateral thigh and covered with damp dressing. The patient recovered acceptable standing and walking functions.

Conclusion: Major chronic soft tissue defects can become covered using NPWT, avoiding amputation.

\section{CHRONIC WOUND SESSION}

\section{Negative pressure therapy in acute and chronic wounds}

\section{S. Goryunov, I. Abramov, B. Chaparian, S. Zhidkikh, A.} Prividencev, M. Egorkin

State Hospital No. 15 n.a O.M. Philatov, Russian National Research Medical University n.a. N.I. Pirogov, Moscow, Russia.

Introduction: Negative pressure wound therapy (NPWT) has been used in our institute to treat 194 patients with acute (91) and chronic (103) wounds. Two representative cases are presented.

Case descriptions: A 29-year-old female had undergone a caesarean and developed necrotizing fasciomyositis complicated by severe anterior abdominal wall sepsis, and was being ventilated. The wound remained unresponsive, with heavy exudation, necrosis and peripheral inflammation. Necretomy was performed followed by continuous NPWT using VivanoTec for 9 days, with Sorbalgon as secondary dressing and $1 \mathrm{~g}$ invanz. The wound completely resolved with a systemic inflammatory response, decreased size and was filled with granulation. Autodermoplasty was performed following the third bandage change. The patient was discharged with complete healing on day 20.

A 68-year-old female had a progressive circular ulcer of the left shin for 1 year, with necrosis and peripheral inflammation. Systemic treatment was $1000 \mathrm{mg}$ prednisolone for 3 days and $1.5 \mathrm{~g}$ meronem, both intravenously. Following debridement, continuous NPWT was initiated, with Atrauman Ag as secondary dressing. Remission of the pyoderma gangrenosum occurred and the wound was covered in granulation tissue after 10 days. Autodermoplasty was performed 5 days later, supported with $40 \mathrm{mg}$ prednisolone and $4 \mathrm{~g}$ sulfasalazine. The patient was discharged 6 days later.

Conclusion: Positive results were obtained in 186 of 194 patients. NPWT significantly optimised acute- and chronicwound treatment through well-controlled exudation, rapid elimination of acute inflammation and stimulation of reparation processes, leading to accelerated wound preparation for plastic surgery and reduced time and costs.

\section{Negative pressure treatment of a diabetic foot chronic wound}

\section{W. Klonowski}

Wojewódzki Szpital Zespolony, Płock, Poland.

Introduction: With over 350 million people in 2011 with diabetes, of whom $2 \%$ annually develop new foot ulceration, of which $8 \%$ require amputation within a year, this represents a major challenge. Negative pressure wound therapy (NPWT) is applied for the local treatment of diabetic foot to support granulation formation, allowing skin grafting, and to stimulate healing. Here we present our first experience using NPWT.

Case description: A 38-year-old male with insulindependent diabetes and arterial hypertension presented with a purulent and gangrenous wound on the right foot sole. Previous systemic treatment included antibiotics, non-steroidal antirheumatic and painkiller, and topical silver-dressing. On day 1 the wound was debrided, Atrauman Ag and a TenderWet dressing pad were applied, and the patient received the antibiotic ertapenem $(1 \times 1.0 \mathrm{~g})$ and intensive insulin therapy. The patient had pain and infection on day 2, which were treated using ertapenem and enoxaparin. The Atrauman Ag and TenderWet dressing were continued. Tissue culture on day 4 indicated bacterial infection, thus antibiotic medication was changed to imipenem and the wound debrided. After 17 days, once authorisation was obtained for this first patient, NPWT treatment was initiated. Good granulation was present on day 36, skin grafting was performed and NPWT was applied continuously at $-60 \mathrm{mmHg}$. By day 65 the wound had completely healed and the patient was discharged the following day without pain or infection, and received insulin, ramipril, simvastatin and Hydrocoll dressing.

Conclusion: NPWT proved more effective than standard local treatment of diabetic foot.

\section{Negative pressure wound therapy combined with different modalities in thoracic surgery \\ C. Halmy}

Burn Unit, Health Centre of the Hungarian Defense Forces, Budapest, Hungary

Introduction: Plastic surgeons have a guideline "reconstructive ladder" of options with increasing complexity to apply to cover a skin defect. This ranges from non-intervention 
by secondary intention, through the simple methods of suturing and grafting to complicated methods using skin flaps. Here we present the most complicated of eight cases, which required multiple methods and was combined with negative pressure wound therapy (NPWT).

Case description: A 41-year-old male had a third-degree open fracture and following a change of the internal and external fixations was transferred for coverage of the wound using viable tissue. As standard for such a wound, a free latissimus flap was initially attempted. However, this was unsuccessful, and NPWT was applied using VivanoTec. Subsequently, the proximal region of the tibia was covered using a muscular flap from the medial side, while for the middle of the bone a fasciocutaneous flap from the lateral side was used. The remainder of the wound was covered with a skin graft. NPWT was reapplied, during which the graft took. After completion of NPWT treatment, the wound had healed and the patient was mobile, and could be discharged from the hospital.

Conclusion: NPWT can be successfully combined with different modalities of reconstructive surgery to close major skin and soft-tissue defects, which in some cases represents a "salvage procedure".

\section{Negative pressure wound therapy in diabetic foot syndrome chronic wounds \\ C. Toth}

KENEZY Hospital, Debrecen, Hungary.

Introduction: We applied negative pressure therapy (NPWT) to stimulate healing in chronic diabetic foot wounds.

Case descriptions: A 47-year-old male with type I diabetes had a large necrotic, pus-producing wound on the left foot and a second large wound on the planar side. Skin, subcutaneous tissue and tendon necrectomy were performed, and the patient received antibiotic treatment. However, it was impossible to save the foot, which was removed by transmetatarsal amputation. Following debridement, continuous NPWT was applied using VivanoTec with Atrauman Ag dressing. The first dressing change was on day 4. A free skin transplant was performed on day 8. NPWT was applied intermittently for 5 days under a silicone layer and the wound healed by day 30 .

A 62-year-old female was readmitted with necrotic fasciitis 2 months after left femoral amputation. The wound underwent necrectomy and debridement. Continuous NWPT was applied. The foam was changed after 4 days, followed by a second NPWT application. Secondary suture wound closure was performed on day 8 .

A 31-year-old female with type II diabetes and a chronic left crural ulcer for 2 years underwent crossectomy, varicectomy and stripping, but failed to heal after 2 months. Continuous NPWT was applied for 4 days, with Atrauman Ag dressing between the foam and wound. A free skin graft was performed on day 5 followed by intermittent NPWT for 4 days under a silicone layer.
Conclusion: All three patients displayed wound healing after NPWT, including stimulation of the skin graft, when applied.

\section{Negative pressure wound therapy treatment of soft tissue wounds}

\section{S. G. Shapovalov, A. S. Pleshkov, A. V. Panov}

FGBU VCERM, St. Petersburg, Russia.

Introduction: We have applied negative pressure wound therapy (NPWT) in the treatment of soft tissue wounds of different origins and types in 15 patients (10 males and 5 females; 7 had diabetes mellitus, 2 obliterative atherosclerosis, 2 were paraplegic and 4 had no concomitant diseases) to stimulate healing, including in combination with grafting and flap surgery. Here we present a representative case.

Case description: A 69-year-old male presented with a recurrent giant postoperative ventral hernia. Initial surgeries had involved resection of an abdominal aortic aneurysm complicated by dissection and abdominal aorta replacement. After 17 months, plastic repair of a ventral hernia was performed using a sieve graft. The patient again had a hernia after 28 months requiring surgical intervention. Recurrent plastic repair using local tissue and prolene mesh was performed. NPWT was applied using VivanoTec in 12 sessions of 2-4 days over a total of 38 days. Sharp dermotension of the wound was performed and NPWT was reapplied. The wound healed and the patient was discharged for outpatient care 52 days after the plastic surgery.

Conclusion: Positive results were obtained in 11 patients (73\%), 3 patients (20\%) improved but treatment was incomplete and in 1 patient (7\%) NPWT was considered ineffective. The mean treatment time was $40 \pm 44$ days, with NPWT applied a mean of $19 \pm 17$ days during hospitalization. Controlled NPWT is a safe and highly effective method in soft wound treatment, in particular in clinical cases with no effective alternative approaches.

\section{Clinical efficacy and cost effectiveness of negative pressure wound therapy in trophic venous ulcer treatment \\ V. N. Obolensky}

SBFEHC City Clinical Hospital No. 13 of the Department of Healthcare of Moscow, Russia; Russian National Research Medical University, Moscow, Russia.

Purpose: A comparative study of trophic venous ulcer (TVU) treatment using negative pressure wound therapy (NPWT) and traditional topical therapy. 
Methods: Twenty-one patients with a TVU $>50 \mathrm{~cm} 2$ were assigned to test $(\mathrm{n}=9)$ and control $(\mathrm{n}=12)$ groups. There were no significant differences in the mean age, varicose vein disease, wound microbiological profile or baseline bacterial count, although the mean wound area was larger in the test $(163.3 \pm 36.2 \mathrm{~cm} 2)$ compared to the control $(118.8$ $\pm 63.8 \mathrm{~cm} 2)$ group. The test group received primary surgical superficial debridement followed by NPWT for several days until sufficient granulation had formed for autodermal transplantation. Atrauman Ag dressing was placed over the graft and a second NPWT was applied. Subsequent treatment involved multilayer compression bandages of Atrauman Ag, Varolast and Putterbinde, changed weekly. The control group underwent traditional topical therapy. Patients were discharged after wound epithelialisation.

Results: The mean inpatient stay before autodermal plastic surgery (APS) was significantly shorter in the test group compared to the control group $(9.6 \pm 1.8$ and $15.7 \pm 0.3$ days, respectively; $\mathrm{p}=0.004$ ) as was the mean impatient stay $(15.1 \pm 1.9$ and $22.8 \pm 3.8$ days, respectively; $\mathrm{p}=0.043)$, with more APS performed in the test group (100\% vs. $25 \%)$. The mean cost per patient in the test and control groups was $1282.03 €$ and $1730.26 €$, respectively.

Conclusion: NPWT improved the outcome and reduced the risk of nosocomial wound infections, treatment duration and costs compared to current TVU treatment.

Negative pressure wound therapy in sacral wounds

\section{P. W. Trzeciak, J. Porzeżynska, D. A. Walczak, K.} Ptasińska, W. Fałek, L. Kozaczek

Department of General Surgery, John Paul II Memorial Hospital, Belchatow, Poland.

Introduction: We present two case studies of negative pressure wound therapy (NPWT) in the treatment of complicated wounds in the sacral region.

Case descriptions: A 62-year-old male following primary treatment for rectal cancer developed wound dehiscence. After reoperation, the patient required 30 days of intensive care for respiratory insufficiency, and developed fascial necrosis. Primary wound treatment was moist gauze and topical octenidine. The wound became a large evisceration. The infection was treated using ertapenem and anitcoagulants, continuous NPWT was applied for 21 days and a hernia support was used. Subsequently, adaptive surgery was performed, with a mesh implantation. The patient was discharged 14 days later.

A 78-year-old male who had previously suffered two strokes, which had required rehabilitation for fine motor skills, developed a fibrinous and necrotic pressure ulcer. The infected ulcer was treated using ciprofloxacin, and continuous NPWT was applied for 21 days using PermaFoam and Atrauman Ag. The patient developed an anal fistula during NPWT, in response to which a stoma was created on the sigmoid colon.
On completion of NPWT, the patient had no infection or pain and was discharged with a control after 2 months.

Conclusion: NPWT may be useful in the conservative management of wound dehiscence, particularly for a severe condition, with better preparation compared to conventional dressing of infected wounds for subsequent surgical treatment. Similarly, NPWT may be applicable to treat entangled wounds in the anorectal area. In both conditions, NPWT will reduce the duration of hospitalisation and treatment costs.

\section{Negative pressure treatment of purulent necrotic lesions in peripheral arterial disease}

\section{V. Zavatsky}

Department of General Surgery, John Paul II Memorial Hospital, Belchatow, Poland.

Introduction: We present a case study of negative pressure wound therapy (NPWT) application to non-responsive, purulent necrotic lesions.

Case description: A 73-year-old male presented with a trophic ulcer in the lower right leg in the vena saphena magna draining area following aortocoronary bypass. A reverse femoro-popliteal bypass graft was performed. Subsequently, trophic ulcers developed on the medial surface of the lower leg, which became larger and more necrotic, compounded by foot and shin pains. The necrotic circular trophic ulcer in the lowerand mid-third of the leg became purulent with perifocal inflammation. A trophic ulcer developed in the calcaneal region. Systemic treatment included antibacterial, anti-inflammatory and desaggregant therapies and substitution transfusion. Local treatment was povidone bandage with iodine and vacuum therapy. Subintimal recanalization of the superficial femoral artery and posterior tibial artery with reentry of the anterior tibial artery was performed. After 4 days, ulcer hydrosurgical debridement was performed. Continuous NPWT was initiated 2 days later for 14 days, with six dressing changes. On day 7 , the calcaneal region trophic ulcer was debrided and NPWT also applied there. The main defect was closed on completion of NPWT using a perforated transplant. Autodermoplastic reconstruction of the calcaneal region was performed and NPWT halted after 6 days. However, the wound deteriorated and NPWT was reapplied for 17 days, when autodermoplastic reconstruction was repeated. There was $99 \%$ skin acceptance without any local inflammation after 16 days and complete epithelialisation after 2 months.

Conclusion: NPWT can be applied to treat non-responsive, purulent necrotic lesions. 